\title{
Prevalence and Antimicrobial Susceptibility Pattern of Salmonella Species Isolated from Human Blood Samples in Robe Hospital, Bale Zone,South East Ethiopia
}

\author{
Jefar Abera* Shani T.John Velmurugan Shanmugam Habtamu Tedila \\ Collage of Natural and computational Sciences Department of Biology (Stream of Medical and Veterinary \\ Microbiology) Madda Walabu University, PO box 247, Bale Robe, Ethiopia
}

\begin{abstract}
The occurrence of Salmonella was a global challenge in the public health and food production sectors. Salmonella infections were one of the major global public health problems. The present study investigated the prevalence and antimicrobial sensitivity of strains of Salmonella species isolated from human blood samples of the patients in and around regions of Robe Hospital during the period of March to June 2016. This study revealed the fact that the prevalence of Salmonella was more common on males $(14.68 \%)$ than the females $(10.42 \%)$. The total prevalence of Salmonella species in Robe Hospital sample were (25.1\%). The age group more commonly prone to this disease ranged from 1-30 followed by 50-60. The Salmonella Species were isolated by using different Selective media such as, Xylose Lysine Deoxycholate (XLD), Brilliant Green Agar (BGA), Macconkey agar and Salmonella Shigella agar (SS). The plates which showed black colonies were characterized using the biochemical analysis and identified as Salmonella species. Different Commercial antibiotics: Chloramphenicol (C), Rifampin(R), Tetracycline (T), Kanamycin (K), Ciprofloxacin (Cip), Ceftriaxone (CRO), Gentamycin (CN), Doxycline(D) and Streptomycin(S)were used to identified the sensitivity of pattern of Salmonella species. The isolated Salmonella species showed more sensitivity to Ciprofloxacin $100 \%$, Ceftriaxone,91\%, Gentamicin 58\% antibiotics. Other antibiotics Doxycyline, Kanamycin, Chloramphenicol, Rifampin, Streptomycin and Tetracycline showed intermediate to complete resistance against tested Salmonella species. The study provides valuable information to agencies and legislators involved in making policy decisions about the use of antimicrobials.
\end{abstract}

Keywords: Antibiotics, Antibiotic sensitivity test, Biochemical test, Prevalence, Salmonella species.

DOI: $10.7176 / \mathrm{JMPB} / 63-05$

Publication date: January $31^{\text {st }} 2020$

\section{INTRODUCTION}

Salmonella was one of the most common infectious diseases of the world for both humans and animals. Food borne infections caused by Salmonella serotypes occurs at high frequency in industrialized nations and developing countries and is an important public health problem worldwide. Typhoid fever (enteric fever) caused by the bacterium Salmonella enteric serovar typhi is an endemic disease in the tropic and sub tropic. The disease is systemic and is often contracted by ingestion of food or water that is contaminated with the pathogen usually from a fecal-oral source. The illness may be mild or severe but sometimes fatal. It is encountered worldwide but is primarily found in developing countries where sanitary conditions are poor (WHO,2000).

There were 16 million annual cases of typhoid fever, 1.3 billion cases of gastroenteritis and 3 million deaths worldwide due to Salmonella species (Bhunia, 2008). It is among the most commonly isolated food borne pathogens associated with fresh fruits and vegetables. In recent years, the incidence of food borne outbreaks caused by the contamination of fresh fruits and vegetables has increased and become a great concern in industrialized countries (Puiet al., 2011).

The Salmonella bacteria are generally transmitted to humans through consumption of mainly contaminated food of animal origin. The contamination is usually caused by the intestinal materials which often contain Salmonella bacteria that pollute the surface of the carcasses during the slaughtering process as a result lead to Salmonella contamination of meat and meat products (Oosterom, 1991). Salmonella cause self-limited gastroenteritis and the more severe forms of systemic typhoid fever,there are two distinct syndromes caused by Salmonella, typhoid and paratyphoid fevers(enteric fevers), and Gastroenteritis/ Salmonellosis (Kasper et al., 2005 and Goburn et al., 2007).

Salmonella infections are capable of producing serious infections that are often food borne and present as gastroenteritis. However, a small percentage of these infections may become invasive and result in bacteremia and extra intestinal disease (Fluit, 2005). The main reservoirs for non-typhoidal Salmonella are animals such as poultry, livestock, pets and reptiles. Salmonella enteric serovar typhi and Salmonella enteric serovar paratyphi colonize only humans and are often acquired through feacally contaminated food or water, a person who has typhoid fever, or from chronic carriers (CIDRAP, 2006). The outcome of Salmonella infections is determined by the host and the status of the bacterium. Whereas, age, genetic and environmental factors mainly determine the 
status of the host, for the bacterium it is determined by virulence factors (Alphons et al., 2005).Serotypes adapted to man, such as Salmonella enteric serovar typhi and Salmonella enteric serovar paratyphi, usually cause severe diseases in humans as a septicemia typhoid syndrome (enteric fever). These serotypes are not usually pathogenic to animals. Serotypes that are highly adapted to animal hosts, such as Salmonella enteric serovar gallinarum (poultry) or Salmonella enteric serovar bortus-ovis (sheep) usually produce very mild symptoms in man (Fluit, 2005).

Ubiquitous serotypes, such as Salmonella enterica serovar enteritidis or Salmonella enterica serovar typhimurium, which affect both man and animals, generally cause gastrointestinal infections usually less severe than enteric fever. About 1,195 outbreaks of Salmonellosis were reported in Brazil in 2007, with $22.6 \%$ of them provoked by the consumption of foods with raw eggs (Wray, 2001). It is estimated that 22 million typhoid cases are reported with 200, 000 deaths in each 3 year. The estimates in Africa are very low because very few people seek medical attention. For example, 59/100, 000 in East Africa and 39/100, 000 cases in Kenya per year (Kariuki et al., 2012).

Since then, an increasing frequency of antibiotic resistance has been reported from all parts of the world, but more so from the developing countries (Samantray, 1997). The uses of chloramphenicol, ampicillin and cotrimoxazole have become infrequent and quinolones have become the first line of treatment of typhoid fever. A more useful definition of MDRST is reserved for strains resistant to all three first-line ant typhoid a antimicrobial agents, namely ampicillin, chloramphenicol and trimethoprim-sulpha methoxazole (Le and Hoffman,1991). Typhoid fever, caused by MDRST, has become a significant cause of morbidity and mortality over recent years. With the emergence of MDRST, fluoroquinolones have gained importance for the treatment of enteric fever in recent years. Knowledge of the prevalence of Salmonella spp. and their antimicrobial susceptibility patterns is of utmost importance in the institution of appropriate antimicrobial therapy. The present investigation is aimed to screen and identify Salmonella species from blood sample collected from symptomatic ascribed and to assess the antibiotic sensitivity of Salmonella species against commercial antibiotic. This study therefore determines the prevalence and antimicrobial sensitivity pattern of Salmonella isolated from human blood samples. The study further helps to increase the knowledge of available Salmonella and may be used to start database of the Salmonella spp. that are prevalent in Robe, Ethiopia. Furthermore, the information obtained from this study on the antimicrobial sensitivity profile of Salmonella spp could be used for successful selection of drugs and treatment of humans. Proper selection of drugs for effective treatment of diseases may also help to reduce the cost in health care.

\section{MATERIALS AND METHODS}

\subsection{Description of the study area}

The study was conducted at Robe Hospital, in Robe city. Robe was the capital city of Bale Zone, Oromia Regional State and South Eastern Ethiopia. Robe city was located at $430 \mathrm{~km}$ south east of Addis Ababa. The area was situated at $7^{\circ} 00^{\prime} \mathrm{N}$ and $39^{\circ} 58^{\prime} \mathrm{E}$ Latitude and longitude respectively. The mean annual rainfall and temperature of $358 \mathrm{~mm}$ and $15.26^{\circ} \mathrm{Crespectively} \mathrm{(Robe} \mathrm{Metrology} \mathrm{department).Total} \mathrm{population} \mathrm{of} \mathrm{Robe} \mathrm{city} \mathrm{is}$ 72,520 (CSA, 2014). As Health office report Bale zone have 1 capital city (Robe), 4 Governmental hospitals and 84 public health centers in the city and the village and 19 medium clinic in Robe (Personal communication with Bale zone Health office).

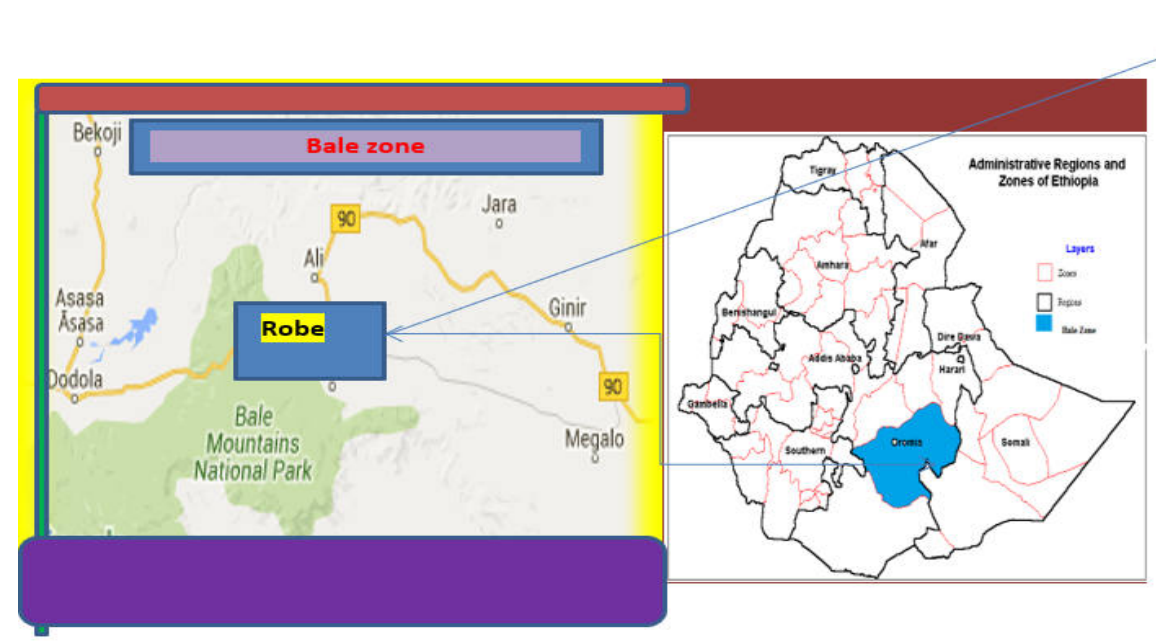

Study area

Figure 1:- The study area Map 


\subsection{Study Design}

The study design was completed based on the patient that visited in Robe Hospital. A cross-section design was used to assess the prevalence of Salmonella patient that visited in Robe Hospital during 2016. Since this study was a laboratory based research, the isolation and identification of Salmonella was done on all blood samples using different techniques in MaddaWalabu University (MDU) laboratory. The purposive sampled was used for all blood samples received during the four month period (March to June 2016).

\subsection{Study Population}

The population of this study was the local peoples of Robe City and surrounding the areas of Robe Hospital that displayed fever, diarrhea and nausea. Totally about 422 patients were recorded within four month in Robe Hospital by collected blood sample from each patient.

\subsection{Sample Size Determination and sampling Technique}

Since the prevalence of Salmonella infections in the study area was not specifically known, the probability of $0.5(50 \%)$ was used for the determination of the sample size for the present study. Therefore, the sample size was determined by following formula as described in Hassan (1991):

$$
\mathbf{n}=\frac{\mathbf{z}^{2} \mathbf{p}(1-\mathbf{p})}{\mathbf{m}^{2}}
$$

Where, $\mathbf{n}$ - sample size,

$\mathbf{z}$ - Confidence level at $95 \%$ (standard value of 1.96),

p - Estimated prevalence of Salmonellas species in the study area,

$\mathbf{m}$ - Margin of error at $5 \%$ (standard value of 0.05 )

$\mathrm{n}=\underline{(1.96)^{2} 0.05(1-0.5)}$

$$
0.05^{2}
$$

$$
\mathbf{n}=\mathbf{3 8 4}
$$

The calculated sample size for the present study was 384 . To minimize errors $10 \%$ of the sample size was added to the normal sample. Therefore, four hundred twenty two (422) individuals blood sample was collected from patient in the present study.

\subsection{Sample collection and transport}

The blood sample were collected from Robe hospital from suspected patients in EDTA tube by medical laboratory technicians and labeled with codes of patients. The labeled tubes were transported to Madda Walabu University Laboratory for further investigation.

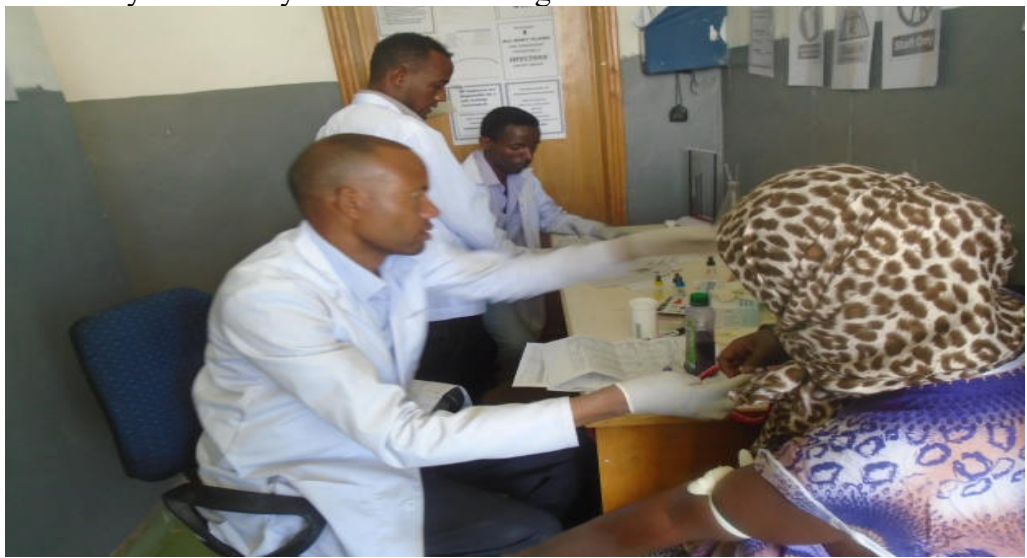

Figure: 2. The blood sample collection procedure

\subsection{Isolation of Salmonella species from blood samples}

The $3 \mathrm{ml}$ of patient's blood was aseptically transferred into Brain Heart Infusion (BHI) broth and incubated at 37 ${ }^{\circ} \mathrm{C}$ for 24 hours. After incubation, a loopful of the enriched cultures of BHI broth was streaked separately onto selective agar plates, Xylose Lysine Deoxycholate (XLD), Brilliant Green Agar (BGA),Macconkey agar and Salmonella Shigella agar (SS). These plates were incubated in an inverted position at $37{ }^{\circ} \mathrm{C} \pm 1{ }^{\circ} \mathrm{C}$ for 18 to $24 \mathrm{~h}$. Following incubation, the black and pink colonies with or without black center on XLD agar, the colorless or opaque-white colonies surrounded by pink or red zone on BGA and black centered colonies on SS agar were identified as Salmonella spp. Such colonies were picked out and sub cultured on Nutrient agar slant and incubated at $37^{\circ} \mathrm{C} \pm 1{ }^{\circ} \mathrm{C}$ for 18 to 24 hours for further biochemical analysis. 


\subsection{Biochemical tests for Salmonella species Identification}

Using a sterile inoculating wire loop, two or more colonies of typical suspicious Salmonella spp were selected from the Nutrient agar slants to perform biochemical confirmation test (Cheesbrough, 2002; Perilla, 2003).The following biochemical tests were performed.

\subsubsection{Triple sugar iron agar (TSI agar)}

The TSI agar slant was inoculated by streaking slant and stabbing the butt with pure culture of typical suspicious Salmonella spp from the Nutrient agar slants. After inoculation the TSI agar was incubated at $37{ }^{\circ} \mathrm{C} \pm 1$ for 18 to 24 hours. The inoculated tubes were caped loosely to maintain aerobic conditions while incubating in order to prevent excessive Hydrogen Sulphide (H2S) production. For interpretation of the TSI results, typical Salmonella cultures show alkaline (red) slants and acid (yellow) butts with gas formation (bubbles) and (in about $90 \%$ of the cases) formation of hydrogen sulfide (blackening of the agar). When lactose-positive Salmonella was isolated the TSI agar slant was yellow. The preliminary confirmation of Salmonella cultures was not based on the results of the TSI agar test only.

\subsubsection{Urea agar test}

The urea agar slant surface was inoculated by streaking the agar slope surface and stabbing the butt with pure culture of typical suspicious Salmonella from the Nutrient agar slants. The urea agar slants were then incubated at $37{ }^{\circ} \mathrm{C} \pm 1{ }^{\circ} \mathrm{C}$ for 18 to 24 hours and the results were interpreted. The positive reaction showed, splitting of urea which liberated ammonia, with changes of the color from phenol red to rose pink, and later to deep cerise (moderate red). The reaction was often apparent after 2 to 4 hours. For a negative reaction, the color of the urea media remained unchanged and has the original color the medium by Salmonella have been observed yellow color and categorized under negative reaction.

\subsubsection{Lysine decarboxylation medium}

Typical suspicious Salmonella spp from the Nutrient agar was inoculated in the L-Lysine decarboxylation medium just below the surface of the liquid medium. The medium was then incubated at $37{ }^{\circ} \mathrm{C} \pm 1{ }^{\circ} \mathrm{C}$ for 18 to 24 hours. Turbidity and a purple color after incubation indicated a positive reaction by which Salmonella also such kind of property. A yellow color indicated a negative reaction.

\subsubsection{Citrate utilization test}

Simmon's citrate slopes were prepared in test tube as recommended by the manufacturer (stored at $2-8^{\circ} \mathrm{C}$ ). The slopes were then stabbed with the suspected organisms and incubated at $37^{\circ} \mathrm{C}$ aerobically for 48 hours (Cheesbrough, 2002). Salmonella was citrate negative as such Simmon's citrate agar slopes remained as green in color and blue color indicates a positive reaction (Bello, 2002).

\subsubsection{Motility Test (using motility agars)}

Motility agar media was prepared and inoculated with the test organisms using a straight inoculating needle making a single stab about $1-2 \mathrm{~cm}$ down into the medium. The motility was examined after incubation at $35-37^{\circ} \mathrm{C}$ for 24 hours. Motility was indicated by the presence of diffuse growth (appearing as coloring of the medium) away from the line of inoculation, all Salmonella species were motile (Cheesbrough, 2002 and Perilla,2003).

\subsubsection{Oxidase test}

There were many method variations to the oxidase test. These include, but were not limited to, the filter paper test, filter paper spot test, direct plate method and test tube method. Soak a small piece of filter paper in $1 \%$ Kovács oxidase reagent and let dry. Use a loop and pick a well-isolated colony from a fresh (18- to 24hourculture) bacterial plate and rub onto treated filter paper. Observe for color changes of microorganisms were oxidase positive when the color changes to dark purple within 5 to 10 seconds. Microorganisms were delayed oxidase positive when the color changes to purple within 60 to 90 seconds. Microorganisms were oxidase negative if the color does not change or it takes longer than 2 minutes as Salmonella also not the color of soaked filter paper.

\subsubsection{Indole test}

The indole test screens for the ability of an organism to degrade the amino acid tryptophan and produce indole. It was used as part of the IMViC (indole, MR-VpCitrate) procedures, a battery of tests designed to distinguish among members of the family Entero bacteriaceae. Inoculate the tube of tryptone broth with a small amount of a pure culture. Incubate at $37^{\circ} \mathrm{C}$ for 24 to 48 hours. To test for indole production, add 5 drops of Kovác's reagent directly to the tube. A positive indole test was indicated by the formation of a pink to red color ("cherryredring") in the reagent layer on top of the medium within seconds of adding the reagent. If a culture was indole negative, the reagent layer was remain yellow or be slightly cloudy also salmonella in case these study observed slightly cloudy and categorized under negative reaction.

\subsubsection{Methyl Red / Voges-Proskauer (MR/VP) test}

This test was used to determine that the fermentation pathway was used to utilize glucose. In the mixed acid fermentation pathway, glucose was fermented and produces several organic acids (lactic, acetic, succinic, and formic acids). The stable production of enough acid to overcome the phosphate buffer was result in a $\mathrm{pH}$ of below 4.4. If the $\mathrm{pH}$ indicator (methyl red) was added to an aliquot of the culture broth and the $\mathrm{pH}$ was below 
4.4, a red color was appearing. If the MR turns yellow, the $\mathrm{pH}$ was above 6.0 and the mixed acid fermentation pathway has not been utilized. The 2,3 butanediol fermentation pathway was ferment glucose and produce a 2,3 butanediol end product instead of organic acids. In order to test this pathway, an aliquot of the MR/VP culture was removed and $\square$-naphthol and $\mathrm{KOH}$ were added. They were shaken together vigorously and set aside for about one hour until the results can be read. The Voges-Proskauer test detects the presence of acetoin, a precursor of 2,3butanediol. If the culture was positive for acetoin, it was turn "brownish-red to pink". If the culture was negative for acetoin, it was turn "brownish-green to yellow".

\subsection{Antibiotic Sensitivity Testing}

The antimicrobial susceptibility testing was done using the agar disc diffusion method as described by the National Committee for Clinical Laboratory Standards (NCLLS, 2002). The pure Salmonella isolates were transferred into nutrient broth (NB) mixed gently until a homogenous suspension was formed. The suspension was incubated at $37^{\circ} \mathrm{C}$ until the turbidity of the suspension becomes adjusted to a $0.5 \mathrm{McFarland}$ standard. The isolates were swabbed gently by sterile cotton swab onto Muller Hinton Agar (MHA) and allowed to dry for half an hour. The antibiotic discs Chloramphenicol (30 mcg), Rifampin (5mcg), Tetracycline (30mcg), Kanamycin (30mcg), Streptomycin (10mcg), Ciprofloxacin (5mcg), Ceftriaxone $(30 \mu \mathrm{g})$, Gentamicin $(10 \mu \mathrm{g})$ and Doxycline $(30 \mu \mathrm{g})$ were aseptically placed over plates of Muller Hinton Agar (MHA) (Haniyeh et al., 2010). The plates were incubated in an upright position at $37^{\circ} \mathrm{C}$ for 24 hours and the zone of inhibition was measured (in mm diameter). The zone of inhibition (sensitive, intermediate and resistant) was interpreted according to (Kirby Bauer et al., 1966, NCCLS, 2002 and Popoff, 2001).

\subsection{Interpretation of zone size}

Inhibition zones produced by each drug was considered into three susceptibility categories namely Sensitive (S), Intermediate (I) and Resistant (R) (NCCLS, 2002) (Table.1).

$\checkmark$ Resistant: A bacteria reported as 'resistant' implies that the infection it has caused was not respond to treatment with the drug to which it is resistant irrespective of dose or site of infection.

$\checkmark$ Intermediate: A bacteria reported as intermediately sensitive suggests that the infection it has caused is likely to respond to treatment when the drug is used in larger doses than normal or when the drug is concentrated at the site of infection. Consideration should be given using other drugs that may provide more optimal therapy.

$\checkmark$ Sensitive (Susceptible):- A bacteria reported as sensitive suggests that the infection it has caused is likely to respond to treatment when the drug is used in normal recommended doses. The standard value for the antibiotic used in these study level was listed below (Table.1).

Table-1: Interpretation standard value of antibiotic used in the study (NCCLS, 2002)

\begin{tabular}{|c|c|c|c|c|}
\hline \multirow[t]{2}{*}{ Antibiotics } & \multirow{2}{*}{$\begin{array}{l}\text { Disc potency } \\
\text { mcg/disc }\end{array}$} & \multicolumn{3}{|c|}{ Diameter of zone of inhibition } \\
\hline & & $\begin{array}{l}\text { Resistance }< \\
\mathbf{m m}(\mathbf{R})\end{array}$ & Intermediate(I)mm & Sensitive $>$ mm $(\mathbf{S})$ \\
\hline Chloramphenicol (C) & 30 & 12 & $13-17$ & 18 \\
\hline Rifampin(R) & 5 & 16 & $17-19$ & 20 \\
\hline Tetracycline(T) & 30 & 14 & $15-18$ & 19 \\
\hline Kanamycin(K) & 30 & 13 & $14-17$ & 18 \\
\hline Streptomycin $(\mathrm{S})$ & 10 & 11 & $12-14$ & 15 \\
\hline Ciprofloxacin(Cip) & 5 & 15 & $16-20$ & 21 \\
\hline Ceftriaxone (CRO) & 30 & 13 & $14-20$ & 21 \\
\hline Gentamicin $(\mathrm{CN})$ & 10 & 12 & $13-14$ & 15 \\
\hline Doxycline(D) & 30 & 12 & $14-20$ & 16 \\
\hline
\end{tabular}

SOURCE: NCCLS (2002)

\subsection{Statistical analysis}

Statistical analysis data were entered in to Microsoft excel spread sheet and analyzed using SPSS window version 20 software.

\subsection{Quality controls}

In order to manage the quality of the work, standard operational procedure were followed during processing of each blood sample. The entire instrument used for sample processing was checked for sterility and proper functioning. For quality control, the strains of Salmonella typhi (MTCC 734) which obtained from Ethiopian Public Health Institute. Completeness of the questionnaire was checked whether necessary information was properly full filled or not. The sterility of prepared media was checked by incubating one of the prepared media 
for 24 hour at $37^{\circ} \mathrm{C}$.

\subsection{Ethical Consideration}

Ethical approval of the study was obtained from the Ethical Review Committee of Bale zone Health center. Official permission was obtained from the Robe Hospital medical director for the collection of blood samples from hospital. The research was done in Applied Microbiology Laboratory of the Department of Biology where the necessary protective wears such as gloves as well as safety cabinets were used to minimize the risk of exposure to human pathogenic bacteria used in this study.

\section{RESULTS}

\subsection{Socio-Demographic Data}

A total of 422 sample from all Robe Hospital was collected for continues four month within the week. The sample was collected at least tree time per-week by using simple random method and structured questionnaire used to assess risk factors associated with Salmonellosis. Of these, the rate of isolation of Salmonella species was significantly higher in pediatric and adult group. The more number of patients were between the age group of $1-$ 30 and followed by 50 to 60 year (Table. 2 ).

Salmonella disease faced worldwide. It was primarily found in developing countries where hygiene conditions was poor (WHO, 2000). As our country was one of the developing country the result indicated that Robe city people was not as much as think for their hygiene spatially male came from other area to Robe Hospital mostly from village area affected with salmonella disease than female. In case of these study as the time of study is on the rain time most of the patient have been came Robe Hospital from these the number of patient came to Robe Hospital have been(59\%) came from urban (Robe city) whereas ( $41 \%$ ) was been came from Rural area from which the sample was been collected for study Salmonella case(Table.2).

Table.2.The data of Socio-Demographic

\begin{tabular}{|l|l|c|}
\hline \multirow{4}{*}{ Age group } & Characteristic & Number (\%) \\
\cline { 2 - 3 } & $1-10$ & $97(23)$ \\
\cline { 2 - 3 } & $11-20$ & $89(21.1)$ \\
\cline { 2 - 3 } & $21-30$ & $60(14.2)$ \\
\cline { 2 - 3 } & $31-40$ & $34(8.1)$ \\
\cline { 2 - 3 } & $41-50$ & $30(7.1)$ \\
\cline { 2 - 3 } Address & $51-60$ & $58(13.7)$ \\
\cline { 2 - 3 } & $>60$ & $54(12.8)$ \\
\cline { 2 - 3 } & Total & $\mathbf{4 2 2 ( 1 0 0 )}$ \\
\hline Patient & Urban & $\mathbf{2 4 9 ( 5 9 )}$ \\
\cline { 2 - 3 } & Male & $102(41)$ \\
\cline { 2 - 3 } & Female & $147(59)$ \\
\cline { 2 - 3 } & Total & $\mathbf{2 4 9 ( 1 0 0 )}$ \\
\cline { 2 - 3 } & Rural & $\mathbf{1 7 3 ( 4 1 )}$ \\
\cline { 2 - 3 } & Male & $136(78.6)$ \\
\cline { 2 - 3 } & Female & $\mathbf{1 7 3 ( 1 0 0 )}$ \\
\cline { 2 - 3 } & Total & \\
\hline
\end{tabular}

\subsection{The prevalence of Salmonella in Robe hospital}

A total of 422 clinically suspected cases of Salmonella patients were selected for the present study. Among the 422 suspected samples, blood cultures positive for Salmonella species was $(25.1 \%)$ and remaining $(74.9 \%)$ were negative. Among the suspected positive salmonella isolates, greater numbers were Male population $(58.5 \%)$ and fewer numbers were Female population (41.5\%). Total prevalence of Salmonella species in Robe Hospital based samples were $25.1 \%$. The male patients consistently remained higher in number than females (Table.3).

Table .3.The Total number of Samples (Prevalence of Salmonella in patients)

\begin{tabular}{|l|l|l|l|}
\hline Sex & Positive No. (\%) & Negative No. (\%) & Total No. (\%) \\
\hline Male & $62(58.5)$ & $203(64.2)$ & $265(62.8)$ \\
\hline Female & $44(41.5)$ & $113(35.8)$ & $157(37.2)$ \\
\hline
\end{tabular}

\subsection{Biochemical Test results}

The test which under talked for Triple sugar iron agar (TSI) test from A yellow butt (acid) and red or pink (alkaline) slope indicates the fermenting of glucose only. Cracks and bubbles in the medium indicate gas production from glucose fermentation by which Salmonella have been follow the characteristics and TSIpositive. In case of Urease Test Salmonella do not change the color of the medium, which was yellow-pink and 
the result was negative. Simon's citrate slopes were prepared in test tube as recommended by the manufacturer (stored at $2-8^{\circ} \mathrm{C}$ ) the blue color slant indicates positive result which was true for Salmonella also. Motility was indicated by the presence of diffuse growth away from the line of inoculation the result indicated that Salmonella was diffused from line of inoculation by which it indicated that positive result. The BCP eventually turns the medium purple in response to the accumulation of cadaverine. Positive tests return to the original purple color sense Salmonella Lysine Decarboxylase test-positive. Salmonella is oxidase negative because it does not change its color with 5-10 seconds. The indole test screens for the ability of an organism to degrade the amino acid tryptophan and produce indole and the result of Salmonella is negative as the color of the medium remains yellow. For MR test the organisms turned yellow color to red indicated positive for Salmonella but In case of Voges-Proskauer- test the culture is negative for acetoin; it was turn "brownish-green to yellow "which the same result with Salmonella(Table.4).

Table.4. Biochemical identification of Salmonella species from blood samples

\begin{tabular}{|l|l|l|}
\hline S.No & Test & Results \\
\hline 1 & Motility & + \\
\hline 2 & Triple sugar Iron test & + \\
\hline 3 & Oxidase & - \\
\hline 4 & Urease & - \\
\hline 5 & Citrate utilization & + \\
\hline 6 & Indole & - \\
\hline 7 & Methyl Red & + \\
\hline 8 & Voges-Proskauer & - \\
\hline 9 & Lysine decarboxylation & + \\
\hline
\end{tabular}

\subsection{Antibiotic Sensitivity test}

Out of 160 samples, 106 Salmonella species showed the characteristics of salmonella after biochemical test and under goes antibiotic sensitivity test by using commercial antibiotics. The isolated Salmonella species showed more sensitivity to Ciprofloxacin (100\%), Ceftriaxone (91\%) and Gentamycin (58\%) antibiotics. Some isolates produced intermediated zone of inhibition against following antibiotics Gentamicin (42\%), Kanamycin and Doxycline (30\%). The resistance was most commonly observed to some of the antibiotics like Tetracycline (100\%),followed by Streptomycin and Rifambin(95\%),Chloramphenicol 90\%,Doxycline 55\% and Kanamycin $40 \%$. Most of the Salmonella isolates showed resistance to two or more antibiotics (Figure.3and Table.5).

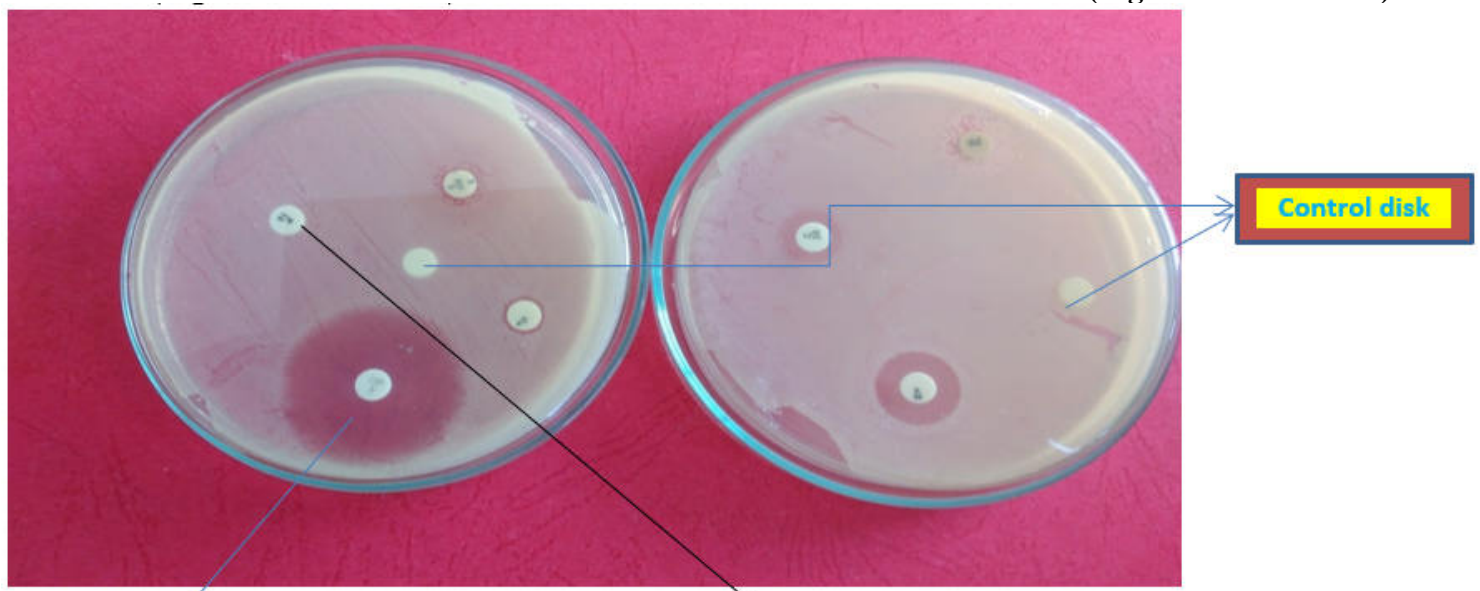

Figure.3: The antibiotic sensitivity of Salmonella species

\section{THE AREA ANTIBIOTIC PUSHED BACTERIA THE DISCS THAT WAS NOT}

\section{PUSHED BACTERIA}

NB: The discs was been put about $\mathbf{1 5} \mathbf{m m}$ from the edge of the plate and no closer than $\mathbf{2 5 m m}$ from disc to disc to controlled the overlap of the inhibition zone of antibiotic zone. No more than $\mathbf{6}$ discs should be applied in $\mathbf{9 0} \mathbf{m m}$ dish. Each disc was been lightly pressed down to ensure its contact with the agar. It was not been moved once in place. 
Table.5: Antibiotic Sensitivity pattern of isolated Salmonella $(\mathrm{n}=106)$

\begin{tabular}{|c|c|c|c|c|c|}
\hline \multirow[b]{2}{*}{ S.No } & \multirow[b]{2}{*}{ Antimicrobial agent } & \multirow[b]{2}{*}{ Strength(mcg) } & \multicolumn{3}{|c|}{ Isolates } \\
\hline & & & $\begin{array}{l}\text { Sensitive } \\
\text { No(\%) }\end{array}$ & $\begin{array}{l}\text { Intermediate } \\
\text { No(\%) }\end{array}$ & Resistance No.(\%) \\
\hline 1 & Chloramphenicol(C) & 30 & $0(0)$ & $11(10)$ & $95(90)$ \\
\hline 2 & Rifampin(R) & 5 & $0(0)$ & $5(5)$ & $101(95)$ \\
\hline 3 & Tetracycline(T) & 30 & $0(0)$ & $0(0)$ & $106(100)$ \\
\hline 4 & Kanamycin(K) & 30 & $32(30)$ & $32(30)$ & $42(40)$ \\
\hline 5 & Streptomycin(S) & 10 & $0(0)$ & $5(5)$ & $101(95)$ \\
\hline 6 & Ciprofloxacin(CIP) & 5 & $106(100)$ & $0(0)$ & $0(0)$ \\
\hline 7 & Ceftriaxone(CRO) & 30 & $97(91)$ & $16(15)$ & $0(0)$ \\
\hline 8 & Gentamicin $(\mathrm{CN})$ & 10 & $62(58)$ & $44(42)$ & $0(0)$ \\
\hline 9 & Doxycline(D) & 30 & $16(15)$ & $32(30)$ & $58(55)$ \\
\hline
\end{tabular}

S-Sensitive, I- Intermediate, R-Resistance

Generally from these study we have been conclude that the antibiotic which was been effective one year was not continually used to controlled the salmonella for more year because the bacteria was been adapted the affecting sit of the antibiotic.

\section{DISCUSSION}

Salmonella is still a significant public health problem in many developing countries. It is a dreaded disease because of its long course and associated complications if not detected and treated early, a leading cause of hospital acquired infections which have been affected male than female in case of present study .This phenomenon is associated with the high isolation rates of Salmonellae and the high prevalence of Salmonellosis among males by (Fashae et al.,2010). The percentage of Salmonella disease among male was $14.68 \%$ where as female was $10.42 \%$. This finding goes in agreement with Females are less likely to be found eating, drinking and defecating outdoors. This is because of culture and religious inclination. This finding is corroborated with Females are less exposed to occupational hazard of farming, related water contact activities, contaminated environment, contaminated food and drink than their males ${ }^{\text {ee }}$ counterpart (Abdullahi et al.,2010). The total prevalence of Salmonella species in Robe Hospital based samples were $25.1 \%$.The risk to Salmonellosis is increased due to the following factors; absence of effective vaccines, modifying hand washing behavior after defecating to control prolonged community out breaks and identifying high risk groups and targeting prevention measures (Perilla, 2003).

The present study results showed that children at the age group of (1-10) are also more commonly prone to Salmonella infections which earlier been documented by (Cajetanetal.,2013) and the adults do not usually predispose themselves to various contaminated areas than young (Mashi,2013). The Salmonella disease affected the age between $(1-30)$ and $(50<)$ deaths due to Salmonella are uncommon, except in the young and old, or the immune suppressed (Heymann,2004). Therefore, it can be concluded that such insusceptibility could reflect on age dependent acquired immunity (Islam et al.,2010).A study done in Ethiopia on the prevalence of Salmonella from beef carcasses in abattoirs found the prevalence rate to be $13.3 \%$ (Dabassa and Bacha, 2012). Another study in Ethiopia found the prevalence rate of Salmonella contamination of minced beef to be $14.4 \%$ (Ejeta et al., 2004).

Identification of Salmonellas pecies was done biochemically by Triple sugar Iron (TSI) test with/out gas or $\mathrm{H}_{2} \mathrm{~S}$ production (Cheesbrough, 2002). The results of motility agar are often difficult to interpret. Generally, the bacteria will move away from the stab mark (are motile) in case of the present study Salmonella has moved away from stab line which was positive (D'Aoust et al., 2007). The Salmonellas pecies was urease negative(Cowan and Steel, 2002),Salmonella is citrate negative as Simmon's citrate agar slopes remained as green in colour and blue colour indicates a positive reaction (Bello, 2002) in the present study Salmonella have changed green color to the blue (positive) and Lysine Decarboxylase test is Positive (Cheesbrough, 2002 and Perilla, 2003). The present result have been almost the same with that of quality control which have been done with standard bacteria Salmonella typhi (MTCC 734) used for more confidential of these study.

The antibiotic sensitivity study, results showed all isolates were highly sensitive to Ciprofloxacin (100\%) and Ceftriaxone (91\%) and this finding is in support with other researches (Adesiyn et al., 1988, Zhang et al., 1998). According to current guidelines Ciprofloxacin and Ceftriaxone is effective for the treatment of salmonellosis (Senthilkumar et al, 2005).In the present study intermediate sensitivity of by Gentamycin (42\%) and by kanamycin and Doxycline $(30 \%$ )was observed. In past study kanamycin and Doxycline antibiotics were used as the most effective drug to control most of Salmonella disease but through the time the bacteria adapted the behavior of that antibiotic and resisted them. However, the subsequent appearance and spread of antibiotic resistance in Salmonella organisms have made many currently available antibiotics ineffective (Kam et al., 
2007).

Various Salmonella serovars resistant to conventional antibiotics such as ampicillin, chloramphenicol, trimethoprim-sulfamethoxazole, and other newer antibiotics (quinolones and extended-spectrum cephalosporins) have been reported with increasing frequency in many areas of the world (Su et al.,2004). Most Salmonella isolates were resistant to amoxicillin, clavulanic acid, chloramphenicol, amoxicillin and co-trimoxazole. Similar results were observed in the present study, Salmonella isolates were resistant to Tetracycline (100\%), followed by Streptomycin and Rifampin (95\%), Chloramphenicol (90\%), Doxycline (55\%) and Kanamycin (40\%).Multidrug resistance (MDR) is still common in Salmonella enterica serovar typhi, although it is declining with increased use of fluroquinolones and cephalosporin for the treatment (Chande et al., 2002, Manchanda et al., 2006).Chloramphenicol was the best antibiotics choose for broad spectrum disease by (CDC-NARMS, 2007). Since the introduction of chloramphenicol in 1948, it has been the drug of choice in the treatment of Salmonella in most parts of the world. But indiscriminate use of the drug and acquisition of plasmid mediated $\mathrm{R}$ factor has led to the development of resistance to Salmonella against this drug(Agarwal et al., 1981). The resistance to tetracycline might be due to the antibiotic being one of the most commonly used antibiotics for animal production,(Senthilkumar et al, 2005).

Initially, reduced use of Streptomycin and chloramphenicol was associated with a decreased prevalence of MDR strains, but recently, continued dependence on ciprofloxacin for the empirical treatment of Salmonella species in Ethiopia and elsewhere has led to the emergence of resistance of Salmonella species to this drug (Saha et al.,1999). In this context of changing the dynamics of resistance to antibiotics, it is imperative for optimal patient care that accurate and early isolation of Salmonella and its antibiotic susceptibility pattern be available to the clinician. Although the conventional method of antibiotic susceptibility testing by disc diffusion method is used to select appropriate antimicrobial drug (Bauer et al.,2006).

Emergence of bacterial resistance to well-known and trusted antibiotics is widely recognized as one of the greatest challenges that physicians face in the management of adult and pediatric infections (Dajani, 2002 and Cohen 1992). In the present study, it was observed that Salmonella species was more prevalent among the tested patients. It was noted that Salmonella was more prevalent in old male and children. Ciprofloxacin was the most effective antimicrobials while Tetracycline was the least susceptible antimicrobial agent in the found in the present study. To prevent Salmonellosis in children and aged individual observation of personnel hygiene and environmental sanitation, cleaning hand with soap/detergent immediately after defecation or after washing child's bottom after defecation and Oral hydration should be encouraged also vaccination or immunization for Salmonellosis should be given good attention (Barrow et al., 2007).

\section{CONCLUSION}

Salmonellosis was transmitted through contamination of food and water sources. The study concluded that the children's and adults age group was more susceptible to Salmonella disease. Finding that the Salmonella isolates in this study were sensitive to ciprofloxacin suggests that this drug should be protected against emergence of resistant strains. The present study revealed that Ciprofloxacin followed by Ceftriaxone was the most effective drug against Salmonella species. The findings of the present study may help improve the prudent use of antimicrobials in Ethiopia and may help direct the proper selection of antimicrobials for the treatment of infections in humans. Similarly, these data may provide important information for future control of important antimicrobial agents used in humans. This study provides valuable information to agencies and legislators involved in making policy decisions about the use of antimicrobials.

\section{REFERENCES}

Abdullahi, M, Olonitola, S. O and Inabo, IH (2010). Isolation of Bacteria associated with diarrhoea among children attending some hospitals in Kano metropolis, Kano state, Nigeria. Bayero Journal of Pure and Applied Sciences;3:10 -15.

Adesiyun,A.A.,Dasuki,M.O and Ibrahim G.A (1988). Occurrence and anti-biograms of Salmonella isolated from slaughter sheep and goats in Zaria, Nigeria and Israel. Journal of Veterinary Medicine; 44:248-255.

Agarwal., K.C., PanHotra,B.R and Mahanta, J.(1981). Typhoid fever due to chloramphenicol resistant S. typhi associated with 'plasmid. Indian J Med Res; 73:484-488.

Alphons, J. A.M., Asten.V.,Jaap, E and Dijk,V. (2005). Mini review: Distribution of "classic" virulence factors among Salmonella. Immunology and Medical Microbiology; 44:251-259.

Barrow,D.K and Boyd, J.S.K. (2007).Laboratory finding in chemical dysentery.Journal of Pathology and Bacteriology; 58:237-241.

Bauer, A.W., Kirby,W.M., Sherris,J.C and Ture, M .(2006). Antimicrobial susceptibility testing by a $A m J$ ClinPathol; 45: 493-496.

Bello,C.S.S. (2002).Laboratory manual for students of medical microbiology.Second edition; 428-430.

Bhunia,A.K.(2008).Biosensors and bio-based methods for the separation and detection of foodborne pathogens. 
Adv Food Nutr Res; 54:41-44.

Cajetan,I.C.I.,Bassey,B.E.,Ikeneche,N.F.,Isu,R.N.,Casmir,A.A.(2013).antimicrobial susceptibility of Salmonella species associated with. British Microbiol: 431-439.

CDC-NARMS.(2007)."National Antimicrobial Resistance Monitoring System.(NARMS): Enteric Bacteria." Retrieved June 14, 2007, from http://www.cdc.gov/narms/.

Chande,C.,Shrikhande, S., Kapale, S., Agrawal, S and Fule, R.P. (2002). Change in antimicrobial resistance pattern of Salmonella typhi in Central India. Indian J Med Res, 115:248-50.

Cheesbrough,M.(2002).District laboratory practice in tropical countries.E.C.B.S edition Combridge University Press ; 2:97-182

CIDRAP.(2006). Centre for Infectious Disease Research and Policy, Academic Health Centre, University of Minnesota. http://www.cidrap.umn.edu/cidrap/contents/fs/food disease /causes /salmoview.html(accessed on $08 / 08 / 2012$ ).

Cohen, M .T. (1992).Typhoid and paratyphoid fever in the tropics. J Trop Med Hyg; 67:1985- 1989.

D'Aoust,J.Y., Sewell, A.M and Greco, P.(2007). Detection of Salmonella in dry foods using refrigerated preenrichment and enrichment broth cultures: summary of collaborative study;77: 1490-1491.

Dabassa, A and Bacha, K. (2012).The Prevalence and Antibiogram of Salmonella and Shigella Isolated from abattoir, Jimma town, South West, Ethiopia. International Journal of Pharmaceutical and iological Research; 3:143 -148.

Dajani,J.T. (2002) Increase in incidence to resistance to ampicillin, chloramphenicol, a trimethoprim in clinical isolates of Salmonella serotype typhimurium with investigation of molecular epidemiology and mechanisms of resistance, J. Med. Microbiol;48:367-374.2002

Ejeta,G., Molla, B., Alemayehu, D. and Muckle, A. (2004).Salmonella serotypes isolated from minced meat beef, mutton and pork in Addis Ababa, Ethiopia. Revue de Medicine Veterinaire; 155 :547 - 551.

Fluit,A.C.(2005).Mini review: Towards more virulent and antibiotic-resistant Salmonella. .Immunology and Medical Microbiology; 43:1-11.

Goburn,B., Grassl,G.A and Finlay,B.B.(2007)."Salmonella, the host and disease: A brief review." Immunol Cell Biol; 85:112-118.

Haniyeh ,K., Seyyed, M., Seyyed, N. and Hussein, M. (2010). Preliminary study on the antibacterial activity of some medicinal plants of Khuzestan (Iran).Asian Pacific Journal of Tropical Medicine; 3: 180-184.

Hassan, T.(1991). Inferential Statistics. In: Handbook of Research Methods in Medicine. Prof. Bankole (ed). Lagos NERDC Press; pp: 167-211.

Heymann, D.(2004).Control of Communicable Diseases Manual. American Public Health Association: 34:675680.

Islam, A., Butler, T., Kabir, I and Alam, N. H. (2010). Treatment of typhoid fever with ceftriaxone for 5 days of chloramphenicol for 14 days: a randomized clinical trial. Antibiotic Agents and Chemotherapy; 37:15721575 .

Kam, K.M., Luey, K.Y., Chiu, A.W., Law, C.P and Leung,S.F.(2007).Molecular characterization of Salmonella entericaserotype typhiisolates by PFGE in Hong Kong, 2000-2004. Foodborne Pathology Diseases;4:4149.

Kariuki,S.,Revathi,G and Kariuki,N.(2012). Invasive multidrug resistant non-yphoidalSalmonella infections in Africa: zoonotic or anthroponotic transmission. Journal of Medical Microbiology;55:585-591.

Kasper,D.L.,Fauci.A.S.,Longo,D.L.,Braunwald,E.,Hauser,S.LandJameson,J.L.(2005). Harrison's Principles of Internal Medicine. NewYork, The McGraw-Hill companies;5: 897-906.

Kirby J., Bauer A. W., Sherris C. and Turck M. (1966).Antibiotic susceptibility testing by a standardized single disk method.American Journal of Clinical Pathology; 45: 493-496.

Le, T.P and Hoffman S.L.(1991).Tropical infectious diseases: principles, pathogens and practice. Philadelphia, PA: Livingstone;pp;277-295.

Manchanda, V., Bhalla.P., Sethi, M and Sharma, V.K. (2006). Treatment of enteric fever in children on the basis of current trends of antimicrobial susceptibility of Salmonella entericaserovartyphiandParatyphi A. Indian J Med Microbiol; 24: 101-6.

Mashi, B. H. (2013). Typhoid fever: a case study of an endemic disorder in Katsina metropolis. Biological and Environmental Sciences Journal for the Tropics;5: 27-30.

National Committee for Clinical Laboratory Standards (NCCLS). (2002). Performance standards for antimicrobial Susceptibility testing; approved standardsM100-S12, Pennsylvania, USA.

Oosterom, J.O.(1991). Epidemiological studies and proposed preventive measures in the fight against human salmonellosis.International Journal of Food Microbiology;12: 41-51.

Perilla,M. J.(2003). Manual for the laboratory identification and antimicrobial testing of bacterial pathogens of public health importance in the developing world.World health organization.Atlanta Georges U.S.A; pp: $133-284$. 
Popoff,M. Y and Le Minor, L.(2005). Antigenic formulas of the Salmonellaserovars, $8^{\text {th }}$ revision. WHO Collaborating Center for Reference and Research on Salmonella;3:43-46.

Pui,C.F., Wong, W.C., Chai,L.C., Lee,H.Y., Tang, J.Y. H., Noorlis, A.,Farinazleen,M. G., Cheah Y. K and Son, R.(2011). Biofilm formation by Salmonella typhi and Salmonella typhimurium on plastic cutting board and its transfer to dragon fruit. International Food Research Journal;18:31-38

Saha,S K, Talukder, S Y, Islam.M and Saha, S.A.(1999). Highly cefriaxone-resistant Salmonella typhi in Bangladesh.Pediatr Infect Dis J; 18:387-400.

Samantray, S. K.(1997). Typhoid fever resistant to furazolidine, Ampicillin, chloramphenicol and cotrimoxazole. Indian J Med Sci; $20 ; 1-3$.

Senthilkumar,B and Prabakaran,B.(2005).Multidrug Resistant Salmonella typhi in Asymptomatic Typhoid Carriers among Food Handlers in Namakkal District, Tamil Nadu. Indian journal of medical micro;23: 9294.

$\mathrm{Su}, \mathrm{L} . \mathrm{H}, \mathrm{Chiu}, \mathrm{C} . \mathrm{H} ., \mathrm{Chu}, \mathrm{C}$ andOu ,J.T.(2004). Antimicrobial resistance in nontyphoidSalmonella serovars: a global challenge. ClinInfect;39:546-551.

World Health Organization.(2000). Household Water Treatment and Safe Storage Following Emergencies and Disasters: South Asia Earthquake and Tsunami. Available at: www.who.int/household_water/en/(accessed on $08 / 08$ / 2012).

Wray,C. and Davies.R.H.(2001). The epidemiology and ecology of Salmonellain meat producing animals.Journal of Dairy Science; 44: 383-427.

Zhang,Y.X., Li, J.Q and Wang, T.G.(1998). Isolation and identification of Salmonella typhimurium and composition of its pathogenicity with Salmonella enteritidis.Chainese Journal of Veterinary Science andTechnology; 28:26-27. 